\title{
Angiopoietin-2 regulated by progesterone induces uterine vascular remodeling during pregnancy
}

\author{
YANG-GYU PARK, JAWUN CHOI and JAE-WON SEOL \\ Biosafety Research Institute and Laboratory of Biochemistry, College of Veterinary Medicine, \\ Jeonbuk National University, Iksan, Jeonbuk 54596, Republic of Korea
}

Received January 9, 2020; Accepted May 4, 2020

DOI: $10.3892 / \mathrm{mmr} .2020 .11185$

\begin{abstract}
During pregnancy, the uterus undergoes intense neovascularization and vascular remodeling to supply oxygen and nutrients to the embryo. During this period, progesterone secreted from the ovary has effects on vascular remodeling in the endometrium and interacts with angiogenic factors. However, the exact mechanism of uterine vascular remodeling during pregnancy is poorly understood. Therefore, the aim of the present study was to investigate the association between angiopoietin-2 (Ang-2), one of the angiopoietins, and intrauterine vessel remodeling during pregnancy, and to determine the effect of progesterone on Ang-2 levels. Changes in Ang-2 expression were observed according to quantitative modification of progesterone using pregnant mice and human uterine microvascular endothelial cells. As a result, Ang-2 was observed mainly in the mesometrial region (MR) of the uterus during the period between implantation and placentation. Furthermore, a substantial amount of Ang-2 also appeared in endothelial cells, particularly of the venous sinus region (VSR). Interestingly, Ang-2 expression was increased by progesterone, whereas estrogen had limited effects. To confirm the association between Ang-2 and progesterone, the function of the progesterone receptor (PR) was inhibited using RU486, a blocker of PR. Ang-2 expression and vascular remodeling of the VSR in the uterus were decreased when the functions of progesterone were inhibited. Overall, the regulation of Ang-2 by progesterone/PR was associated with vascular remodeling in the VSR during pregnancy. The present study proposed a
\end{abstract}

Correspondence to: Dr Jae-Won Seol, Biosafety Research Institute and Laboratory of Biochemistry, College of Veterinary Medicine, Jeonbuk National University, Gobongro 79, Iksan, Jeonbuk 54596, Republic of Korea

E-mail: jwsseol@jbnu.ac.kr

Abbreviations: HUtMEC, human uterine microvascular endothelial cell; Ang-2, angiopoietin-2; PR, progesterone receptor; MR, mesometrial region; AMR, anti-mesometrial region; VSR; venous sinus region; VEGFs, vascular endothelial growth factors; DPC, day post coitum

Key words: angiopoietin-2, vascular remodeling, pregnant uterus, progesterone, human uterine microvascular endothelial cell solution to prevent pregnancy failure due to a lack of vascularity in the uterus in advance.

\section{Introduction}

Each organ contains blood vessels that undergo neovascularization and vascular remodeling processes for the organ's growth. To understand the regulation of neovascularization and vascular remodeling for each organ, it is necessary to regulate these processes (1). The uterus is a unique organ; it undergoes extensive neovascularization and vessel degeneration during the menstrual cycle, differing from typical neovascularization, which occurs mostly during premenstrual and postmenopausal stages of life. During early pregnancy, the embryo rapidly develops in the uterus; the uterus supplies sufficient oxygen and nutrients for neovascularization and vascular remodeling until the placenta becomes structurally complete and functional $(2,3)$.

Furthermore, in early pregnancy, the decidua supplies a vascular network for the developing embryo before placentation (4). Decidualization of the uterine endometrium involves dramatic differentiation of the uterine tissue, including morphological and functional transformations $(5,6)$. However, during this process, a lack of vascularity in the decidua leads to early abortion and preeclampsia via nutritional deficiency (7). It is fair to say that the uterus requires profuse vascularity in the decidua to prevent pregnancy failure. Endometrium formation resulting from decidualization is termed 'decidual angiogenesis', and involves the impressive development of uterine neovascularization; which includes angiogenesis, vasculogenesis, arteriogenesis - and vascular remodeling stimulated by steroid hormones (8-10).

The steroid hormones released from the ovaries stimulate vascular remodeling and uterine neovascularization, which are necessary for successful pregnancy. Progesterone and estrogen are representative hormones which bind to the progesterone receptor (PR) and estrogen receptor (ER), respectively, and cooperate to regulate decidua formation during early pregnancy $(11,12)$. In addition, progesterone promotes decidual angiogenesis during this period via the vascular endothelial growth factor-A/vascular endothelial growth factore receptore-2 (VEGF-A/VEGFR-2) system. Furthermore, progesterone and estrogen further regulate the induction of angiogenesis in the uterus $(1,13)$. 
Angiogenesis, one of the essential processes in blood vessel formation, represents the development of new branching vessels from existing vascular networks and is operated by endothelial cells, the main components of blood vessels (14). The phenomenon is essential for embryonic growth, wound healing in recovery of adults, and the menstrual cycle in thickening the uterus (15). Angiogenesis also contributes to inflammatory disease and tumor growth. In some cases, inappropriate angiogenesis may result in ischemia (16). Given the variety of functions it performs, vascular remodeling, which results from angiogenesis of the uterus, is essential for successful pregnancy. Angiogenesis and vascular remodeling are thought to be regulated by the cooperative interaction between several angiogenic factors (17). There are two major angiogenic factors and their respective responsive receptors: VEGF and the receptor VEGF-R, and angiopoietin and the Tie receptor, which regulate both neovascularization, including vasculogenesis and angiogenesis, and vascular remodeling, including enlargement and blood network formation, in the uterus (18-20).

The VEGF, one of the major angiogenic factors of vascular regulator in the endometrium, increases endothelial cells' proliferation, permeability, and migration (21). Another vascular growth factor, Angiopoietin-1 (Ang-1), increases the recruitment of endothelial cells with pericytes and vascular smooth muscle cells to remodel newly formed blood vessels, stimulating and stabilizing their maturation (22). Angiopoietin-2 (Ang-2), as an antagonist of Ang-1, plays an important role alongside VEGF, as a regulator of vascular remodeling, to migrate and proliferate endothelial cells. The Ang-1/Ang-2 ratio is inversely associated with blood vessel destabilization, a prerequisite for new blood vessel formation (23). During angiogenesis, Ang-2 binds to its receptor named Tie-2, competitively with Ang-1 (24). Recently, many studies have proved that Ang-2 holds a crucial role in female reproduction (25). Overexpression of Ang-2 in mice resulted in embryonic fatality in consequence to failure of angiogenesis (26). Interestingly, Ang-2 is initially expressed in the ovaries and later, during early pregnancy, in the uterus and placenta (23).

Previous research has shown that progesterone governs uterine angiogenesis and vascular remodeling via VEGF-A/ VEGFR-2 signaling, especially in the anti-mesometrial region (AMR), where the embryo resides during pregnancy (1). However, the functional role of spatiotemporal-localized Ang-2 expression in the pregnancy uterus is not yet fully understood. In our study, we hypothesized that spatiotemporal changes are focused on the mesometrial region (MR) of the uterus because decidual development and vascular remodeling are both developed by Ang-2 which is regulated by progesterone during early pregnancy. To examine the relationship between Ang-2 and progesterone, we underwent in vitro and in vivo experiments. Consequently, our results supported our hypothesis that Ang-2 regulated by progesterone is a key regulator of vascular remodeling in the uterus during pregnancy.

\section{Materials and methods}

Mice. C57BL/6 mice aged 8 to 10 weeks were used for this study and female mice were mated with adult male mice.
Identification of a vaginal plug the following morning was interpreted as successful mating, and designated 0.5 day post coitum (dpc). Ang- $2^{+/ L a c Z}$ mice were transferred and bred in our pathogen-free animal facilities. The Specific pathogen-free (SPF) C57BL/6J mice were all given ad libitum access to standard diet (PMI Lab diet) and water. All animal experiments were performed following approval from the Institutional Animal Care and Use Committees (IACUC) of Jeonbuk National University.

Histological analysis. Mice were sacrificed using the cervical dislocation method on the indicated days. Segments of the uterus containing implanted embryos were fixed in $4 \%$ paraformaldehyde (Biosesang; cat. no. PC2031) for $4 \mathrm{~h}$, followed by overnight dehydration in $20 \%$ sucrose solution. Dehydrated samples were embedded with tissue freezing medium (Scigen; cat. no. 4586) and the frozen blocks cut into $20 \mu \mathrm{m}$ sections.

Samples were blocked with 5\% donkey serum (Jackson ImmunoResearch; cat. no. 017-000-121) or goat serum (Jackson ImmunoResearch; cat. no. 005-000-121) in PBST (0.03\% Triton $\mathrm{X}-100$ in PBS) and then incubated for $4 \mathrm{~h}$ at room temperature (RT) with the following primary antibodies: anti-CD31 (hamster monoclonal, Millipore; cat. no. MAB1398Z), antiAng-2 (rabbit polyclonal, Proteintech TM; cat. no. 24613-1-AP), anti-PR (rabbit polyclonal, Cell signaling; cat. no. 8757), and anti-Tie-2 (mouse monoclonal, Abcam; cat. no. ab24859). After several washes, the samples were incubated for $2 \mathrm{~h}$ at RT with the following secondary antibodies: $\mathrm{Cy} 3$-conjugated anti-hamster IgG (Jackson ImmunoResearch; cat. no. 127-165160), and Cy3- or FITC-conjugated anti-rabbit IgG (Jackson ImmunoResearch; cat. no. 711-165-152 or cat. no. 111-095-003). Nuclei were stained with 4',6-diamidino-2-phenylindole (Enzo; cat. no. BML-AP402). Afterward, the samples were mounted in fluorescent mounting medium (DAKO; cat. no. S3023).

To examine $\beta$-galactosidase activity, the cryo-sections were incubated with a staining solution $[2 \mathrm{mM}$ magnesium chloride, $5 \mathrm{mM}$ potassium ferricyanide, $5 \mathrm{mM}$ potassium ferrocyanide and $1 \mathrm{mg} / \mathrm{ml}$ 4-chloro-5-bromo-3-indolyl- $\beta$-D-galactopyranoside (X-gal) in PBS] at $37^{\circ} \mathrm{C}$ for $24 \mathrm{~h}$. Immunofluorescent images and $\beta$-gal activity were acquired using a Zeiss LSM510 confocal fluorescence microscope (Carl Zeiss) and a microscope equipped with a CCD camera (Carl Zeiss).

Detection of Ang-2 expression by reverse transcription $(R T)-q P C R$. Total RNA was extracted from the uterus using TRIzol ${ }^{\circledR}$ Reagent (Invitrogen; cat. no. 15596018) according to the manufacturer's instructions. The RNA concentration was measured using NanoDrop 2000 (Thermo Fisher Scientific, Inc.). The RNA $(2 \mu \mathrm{g})$ was reverse transcribed into cDNA using SuperScript II Reverse Transcriptase (Invitrogen; cat. no. 18064071). RT-qPCR was carried out using the following conditions: preheating for $5 \mathrm{~min}$ at temperature $95^{\circ} \mathrm{C}$; and then repeating 32 cycles in temperature $95^{\circ} \mathrm{C}$ for $20 \mathrm{sec}$ and $30 \mathrm{sec}$ at $59^{\circ} \mathrm{C}$. The primer sequences were as follows: (1) Ang-2, Foward; 5'-GGATCTGGGGAGAGAGGAAC-3', Reverse; 5'- CTCTGCACCGAGTCATCGTA -3'. (2) GAPDH, Forward; 5'-ACCACAGTCCATGCCATCAC-3', Reverse; 5'-TCCACCACCCTGTTGCTGTA-3'. The PCR products were loaded onto a $1.5 \%$ agarose gel containing Loading 
STAR nucleic acid dye (6X, Dynebio; cat. no. A750), electrophoresed, and photographed using a Fusion FX7 acquisition system (Vilbert Lourmat). The band was semi-quantified using Quantity One software (v4.6.2; Bio-Rad Laboratories, Inc.) with GAPDH as the loading controls.

Cell culture. Human uterine microvascular endothelial cells (HUtMEC) purchased from Lonza Group, Ltd., cat. no. CC-2564) were grown in endothelial cell growth medium (EGM-2MV BulletKit; cat. no. CC-3202) with 5\% fetal bovine serum (FBS) and used at passage 3-4 in all the experiments. Cell were incubated at 37 with $5 \% \mathrm{CO}_{2}$. To examine the change of Ang-2 expression in cultured HUtMEC due to progesterone, the cells were starved for $6 \mathrm{~h}$ and then treated with progesterone and estrogen $(10 \mu \mathrm{M})$ for $24 \mathrm{~h}$.

Immunocytochemistry (ICC) analysis. HUtMEC were cultured on glass slides, fixed with cold acetone, and blocked by $5 \%$ FBS in PBST. They were incubated with anti-Ang-2 (rabbit polyclonal; proteintech TM; cat. no. 24613-1-AP) and anti-VE-cadherin (rabbit polyclonal, Cell Signaling; cat. no. 2500$)$ at $4^{\circ} \mathrm{C}$. Cells were incubated with anti-rabbit IgG conjugated with Alexa Fluor ${ }^{\circledR} 546$ (Abcam; cat. no. ab60317) and anti-human Ig G conjugated with Alexa Fluor ${ }^{\circledR} 488$ (Abcam; cat. no. ab150129). Nuclei were stained with 4',6-diamidino2-phenylindole phenylindole (Enzo; cat. no. BML-AP402). Afterward, the samples were mounted in fluorescent mounting medium (DAKO; cat. no. S3023) and immunofluorescent images were acquired using a Zeiss LSM510 confocal fluorescence microscope (Carl Zeiss).

Western blot analysis. The uterus tissues and cells were homogenized in ice-cold RIPA buffer $(150 \mathrm{mM} \mathrm{NaCl}$, $1 \%$ Triton $\mathrm{X}-100,1 \%$ Sodium deoxycholate, $0.1 \%$ SDS, 50 mM Tris- $\mathrm{HCl}$ pH 7.5, 02 mM EDTA pH 8, Biosesang; cat. no. R2002) on the indicated days. Total protein was quantified using a bicinchoninic acid assay kit (Thermo Fisher Scientific, Inc.; cat. no. 23225). Equal amounts of protein (40 $\mu \mathrm{g}$ per lane) were separated on an 8 12\% SDS-PAGE gel by $120 \mathrm{~V}$ for 90 min in $1 \mathrm{X}$ running buffer and transferred to nitrocellulose (NC) membranes (EMD Millipore; cat. no. 66485). Each NC membrane was blocked in Tris-buffered saline/Tween (TBST) with $5 \%$ non-fat milk for 60 min with gently shaking at room temperature (RT) and then incubated with the designated primary antibodies $(1: 1,000)$ overnight at $4^{\circ} \mathrm{C}$. The primary antibodies used were as follows: anti-angiopoietin-2 (polyclonal, Proteintech; cat. no. 24613-1-AP), anti-progesterone receptor (rabbit polyclonal, Cell Signaling; cat. no. 8757), and anti- $\beta$-actin antibody (rabbit polyclonal, Sigma-Aldrich; cat. no. A2066). Each NC membrane was washed three times in TBST for 10 min per wash and then incubated with the 1:2,000 goat anti-rabbit IgG (Enzo; cat. no. ADI-SAB-300) or goat anti-mouse IgG (Enzo; cat. no. ADI-SAB-100) in TBST with $5 \%$ non-fat milk for $1 \mathrm{~h}$ at RT. The membranes were washed three times in TBST for 10 min per wash and visualized by horseradish peroxidease (HRP) substrate (Enzo; cat. no. ADI-SAB-300-J) using a Fusion FX 7 (Vilber). The band was semi-quantified using Quantity One software (v4.6.2; Bio-Rad Laboratories, Inc.) with $\beta$-actin as the loading controls.
Statistical analysis. Values are presented as mean \pm standard deviation (SD). Significant differences between means were determined by unpaired Student's t-tests or analysis of variance with one-way and two-way ANOVA followed by the Student-Newman-Keuls test or Bonferroni post hoc test. All statistical analysis was performed using the GraphPad Prism software. $\mathrm{P}<0.05$ was considered to indicate a statistically significant difference.

\section{Results}

Ang-2 expressed in the uterine endometrium during early pregnancy, mainly in $C D 31^{+}$blood vessels. We analyzed Ang-2 expression to investigate the role of Ang-2 in early pregnancy (from $4.5 \mathrm{dpc}$ to $8.5 \mathrm{dpc}$ ). Over time, Ang-2 expression increased remarkably around the embryo and spatiotemporal area in the MR, focusing in the venous sinus region (VSR) from implantation until placentation (Fig. 1A). Protein and mRNA expression levels of Ang-2 also rose in the pregnant uterus (Fig. 1B-D). In the 8.5 dpc uterus, Ang-2 protein expression was 2.7-fold higher that of the $4.5 \mathrm{dpc}$ uterus, and Ang-2 mRNA expression was 4.2-fold higher that of the estrus non pregnant mice. We further examined regional Ang-2 expression in early pregnancy through X-gal staining, using Ang-2-LacZ reporter (Ang-2 ${ }^{+/ L a c Z}$ ) mice at 6.5, 8.5 and $10.5 \mathrm{dpc}$. Endothelial cells consisting of blood vessels' lumen in VSR gave off a green color in the presence of Ang-2 to confirm these results (Fig. 2A). During the post-implantation period, expressed Ang-2 was localized on the VSR in the MR. These results indicate that Ang-2 is associated with vascular remodeling and is mainly expressed in CD31 positive blood vessels in the MR. In addition, we performed co-staining of CD31 and Ang-2 via immunofluorescence (IF) method in the $5.5 \mathrm{dpc}$ uterus when the Ang-2 expression initiated to figure out the relationship between endothelial cells and Ang-2. We observed an overlapped color as a result of co-stain red (CD31) and green (Ang-2). The result of stain showed us that Ang-2 was mainly expressed in CD31 positive blood vessels (Fig. 2B). Vascular remodeling of blood vessels regulated by angiogenesis factors is associated with the Tie-2 receptor (27). Therefore, Tie- 2 and Ang-2 expressed in the uterus during early pregnancy were observed. As a result, it was confirmed that Tie- 2 was also expressed in the $\mathrm{CD} 31^{+}$blood vessel that was expressing Ang-2 (Fig. 2C). Through these results, we were able to predict that Ang-2 could be involved in intravascular vascular remodeling via the Tie-2 receptor inside the uterus.

Expression of Ang-2 is regulated by progesterone. In previous studies, it was found that vascular remodeling inside the uterus is regulated by progesterone (1). In addition, vascular remodeling by progesterone occurs via the PR, which is a receptor for progesterone. Based on these results, we expected that Ang-2 expressed in the uterus may be related to progesterone. To confirm this hypothesis, the expression regions of the PR and Ang-2 were compared. As a result, it was confirmed that PR was also expressed at the region where Ang-2 was expressed (Fig. 3A). Ang-2 expression with progesterone treatment in vitro using HUtMEC confirmed the in vivo data. Progesterone treatment increased Ang-2 levels in HUtMEC in comparison to untreated cells, the control, by 1.6-fold. 
A
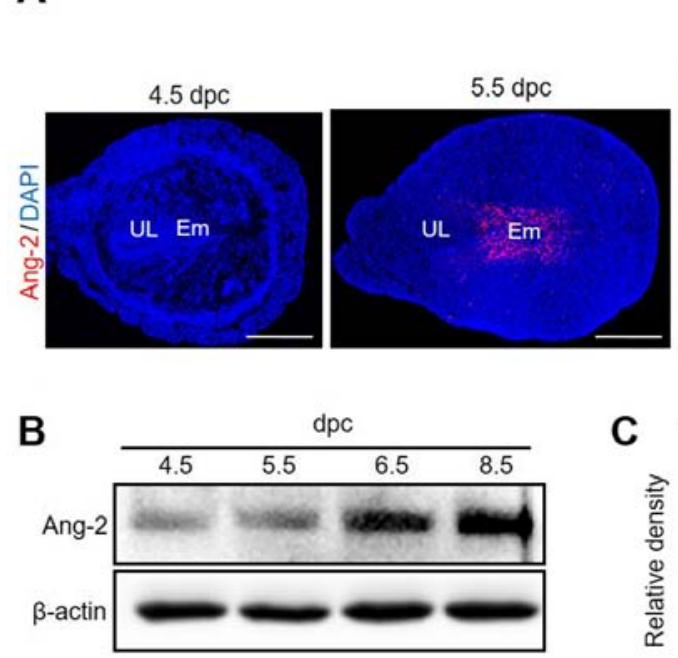

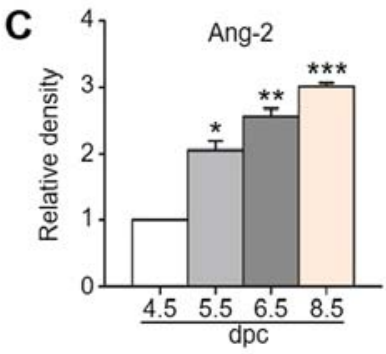

D

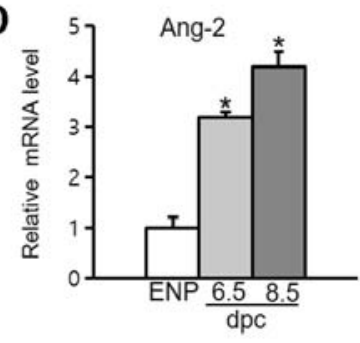

Figure 1. Ang-2 expression in the uterus during pregnancy. (A) Image showing Ang-2 expression in mouse uteri during early pregnancy at 4.5, 5.5, 6.5 and $8.5 \mathrm{dpc}$. Scale bar, $500 \mu \mathrm{m}$. (B) Protein expression levels and (C) semi-quantitative analysis of Ang-2 in the uteri at 4.5, 5.5, 6.5 and 8.5 dpc were measured by western blotting. Loading of similar amounts of protein for each sample was verified by a similar intensity of $\beta$-actin signal. Data are presented as the mean \pm SD from three independent experiments. ${ }^{*} \mathrm{P}<0.05,{ }^{* *} \mathrm{P}<0.01$ and ${ }^{* * *} \mathrm{P}<0.001$ vs. $4.5 \mathrm{dpc}$. (D) Ang-2 mRNA expression in the uteri of ENP, and at 6.5 and $8.5 \mathrm{dpc}$. Data is presented as relative fold change compared with the levels of ENP after normalization to GAPDH. $n=4$ in each group. "P<0.02 vs. ENP. Ang-2, angiopoietin-2; dpc, days post coitum; UL, uterine lumen; Em, embryo; ENP, estrous of non-pregnancy.

A

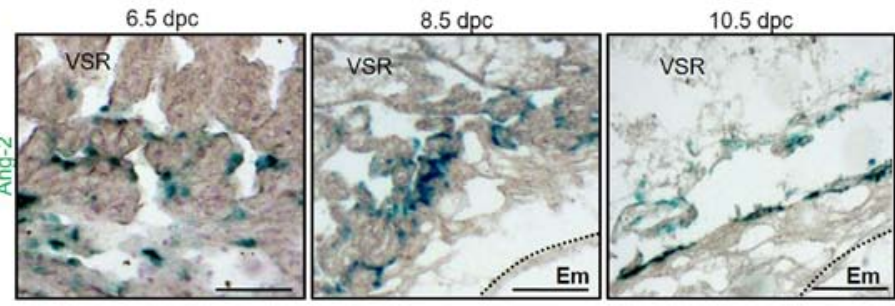

B
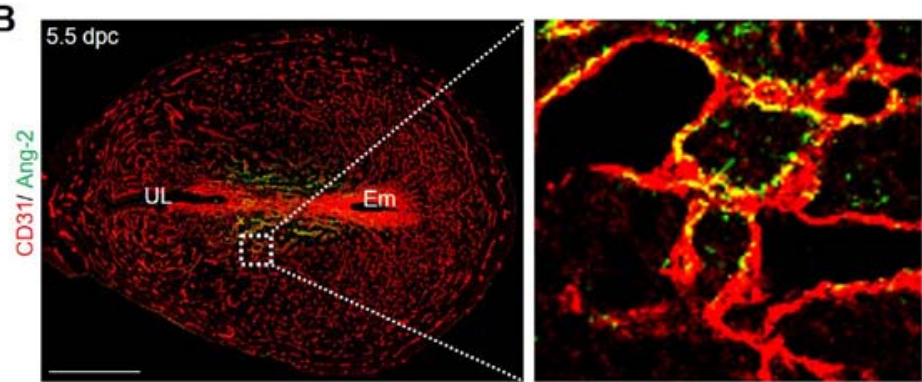

C

CD31/Tie2/Ang2

$\mathrm{CD} 31$
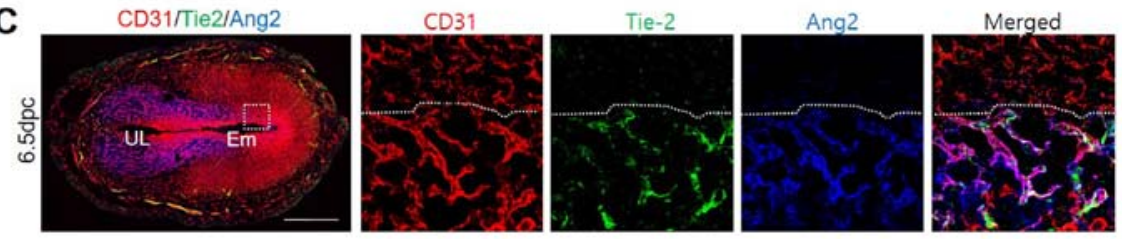

Figure 2. Ang-2 expression around vascular endothelial cells. (A) Image showing Ang-2 expression in the VSR of Ang-2+/LacZ mice at 6.5, 8.5 and 10.5 dpc. The tissues were counterstained with hematoxylin following X-gal staining. Scale bar, $500 \mu \mathrm{m}$. (B) Image showing CD31 ${ }^{+}$BVs and Ang-2 $2^{+}$cells in the uterus at 5.5 dpc. Scale bar, $500 \mu \mathrm{m}$. The section in the square is magnified on the right. (C) Image showing CD31 $\mathrm{BVs}$ and Ang- $2^{+}$and Tie- $2^{+}$cells in the uterus at $6.5 \mathrm{dpc}$. Scale bar, $500 \mu \mathrm{m}$. Ang-2, angiopoietin-2; BVs, blood vessels; dpc, days post coitum; UL, uterine lumen; VSR, venous sinus region; Em, embryo.

However, estrogen treatment was not much different to the control. In addition, there was no difference between treatment with both progesterone and estrogen in HUtMEC and treat- ment with only progesterone (Fig. 3B and C). We assumed that progesterone known as a pregnancy hormone mainly controls Ang-2 in HUtMEC. Moreover, progesterone-treated HUtMEC 
A

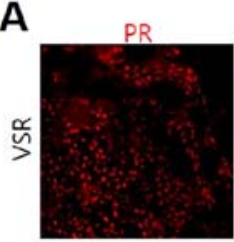

B
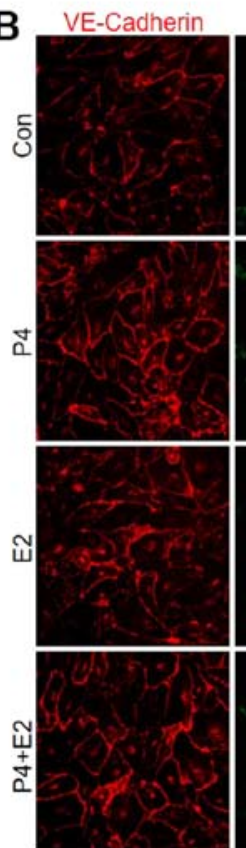

Ang-2

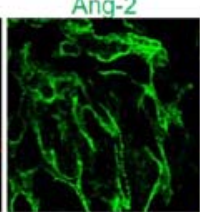

Ang-2
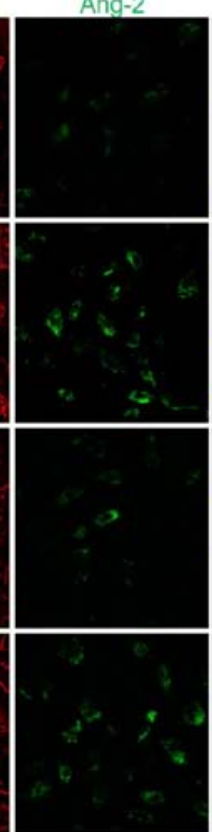

DAPI

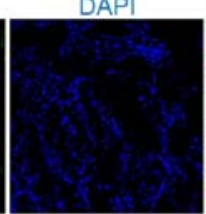

DAPI
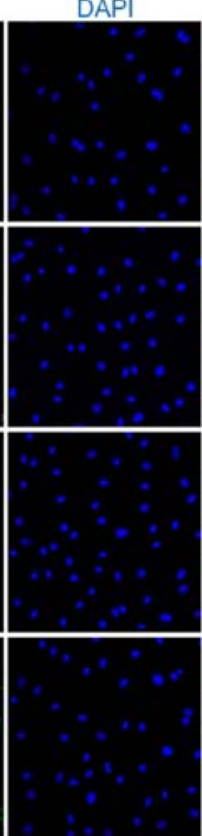

Merged

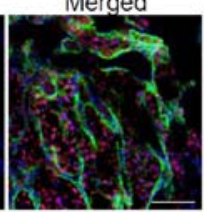

Merged

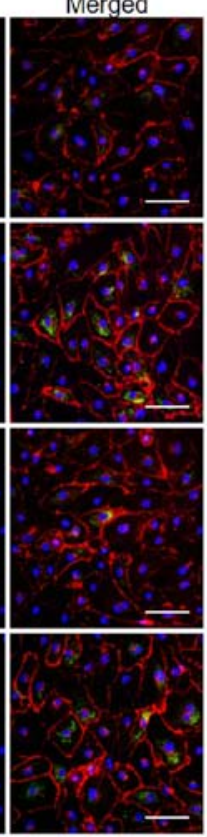

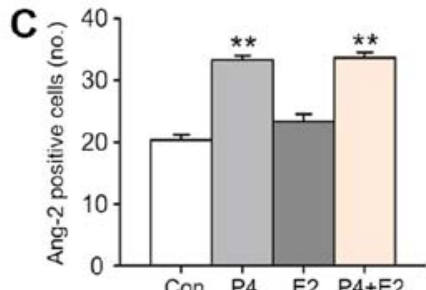

D
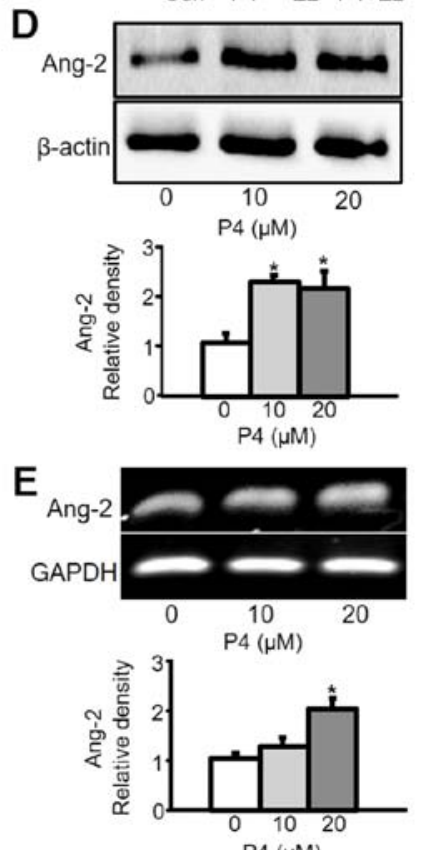

Figure 3. Ang-2 expression is increased by progesterone treatment in HUtMECs. (A) Image showing PR ${ }^{+}$region and Ang- $2^{+}$cells in the uterus at 6.5 days post coitum. Scale bar, $100 \mu \mathrm{m}$. (B) Immunofluorescence staining of VE-Cadherin and Ang-2 in HUtMECs treated with Con, P4 (10 $\mu \mathrm{M})$, E2 (10 $\mu \mathrm{M})$ and $\mathrm{P} 4+\mathrm{E} 2$ (each $10 \mu \mathrm{M})$. Nuclei were counterstained with DAPI (blue). Scale bar, $100 \mu \mathrm{m}$. (C) Comparisons of Ang-2 ${ }^{+}$HUtMECs treated with Con, P4, E2, $\mathrm{P} 4+\mathrm{E} 2(10 \mu \mathrm{M}) .{ }^{* *} \mathrm{P}<0.01$ vs. Con. (D) Comparisons of Ang-2 protein expression levels and relative density (lower graph) in HUtMECs treated with Con and P4 (10 and $20 \mu \mathrm{M})$. Loading of similar amounts of protein for each sample was verified by a similar intensity of $\beta$-actin, and the relative expression levels of Ang-2 after normalization to $\beta$-actin are shown. Three independent experiments exhibited similar results. ${ }^{*} \mathrm{P}<0.02$ vs. Con. (E) Semi-quantitative reverse transcription-PCR showing mRNA levels of Ang-2 in HUtMECs treated with Con and P4 (10 and $20 \mu \mathrm{M})$. The lower graph presents the relative fold change of the levels of Ang-2 after normalization to GAPDH. $n=5$ in each group. * P<0.02 vs. Con. Ang-2, angiopoietin-2; Con, control; E2, estrogen; HUtMECs, human uterine microvascular endothelial cells; P4, progesterone; PR, progesterone receptor; VSR, venous sinus region.

displayed higher Ang-2 protein and mRNA expression than untreated cells (Fig. 3D and E).

Expression of Ang-2 related with PR in pregnancy uterus. Through previous results, we recognized progesterone controlled the expression of Ang-2 in HUtMEC, so we assumed that vascular remodeling operated by Ang-2 regulated its expression in the presence of the progesterone receptor, which binds to progesterone, exists in HUtMEC. To test the hypothesis, we performed western blotting and ICC to discover the progesterone receptor via progesterone treatment. As a result, it was confirmed that PR was expressed regardless of the concentration of the progesterone in HUtMEC (Fig. 4A). Also, expression of PR and Ang-2 was observed in HUtMEC (Fig. 4B). Therefore, Ang-2 in the uterus is expressed by progesterone, and the expression of Ang-2 proves that progesterone is involved via the PR. To clarify the relationship between progesterone and Ang-2, the function of the progesterone receptor was blocked using RU486 ( $8 \mathrm{mg} / \mathrm{kg})$. As a result, it was confirmed that the remodeling of blood vessels inside the uterus was significantly reduced by RU486, and indeed, the expression of Ang-2 was also decreased by RU486 (Fig. 5A and C). Compared to the control groups, treatment with RU486 dramatically decreased the number of blood vessels with diameter of over $300 \mu \mathrm{m}$. Consequently, the number of blood vessels diameter between 100-300 $\mu \mathrm{m}$ increased significantly following RU486 treatment (Fig. 5B). This showed that vascular remodeling is inhibited by RU486 as a pharmacological blockade of PR. Moreover, it was confirmed that Ang-2 expressed in vascular endothelial cells also decreases by the PR blockade (Fig. 5C). Through these results, it was confirmed that when the function of the PR is blocked using RU486, the intrauterine vascular remodeling regulated by the progesterone decreases.

\section{Discussion}

The female uterus experiences cyclic changes from menstrual periods and pregnancy, times of extraordinary neovascularization and vascular remodeling (28). Especially during pregnancy, the maternal body increases the volume of blood towards the uterus to supply oxygen and nutrients for embryo development. Blood vessels in the uterus undergo drastic 

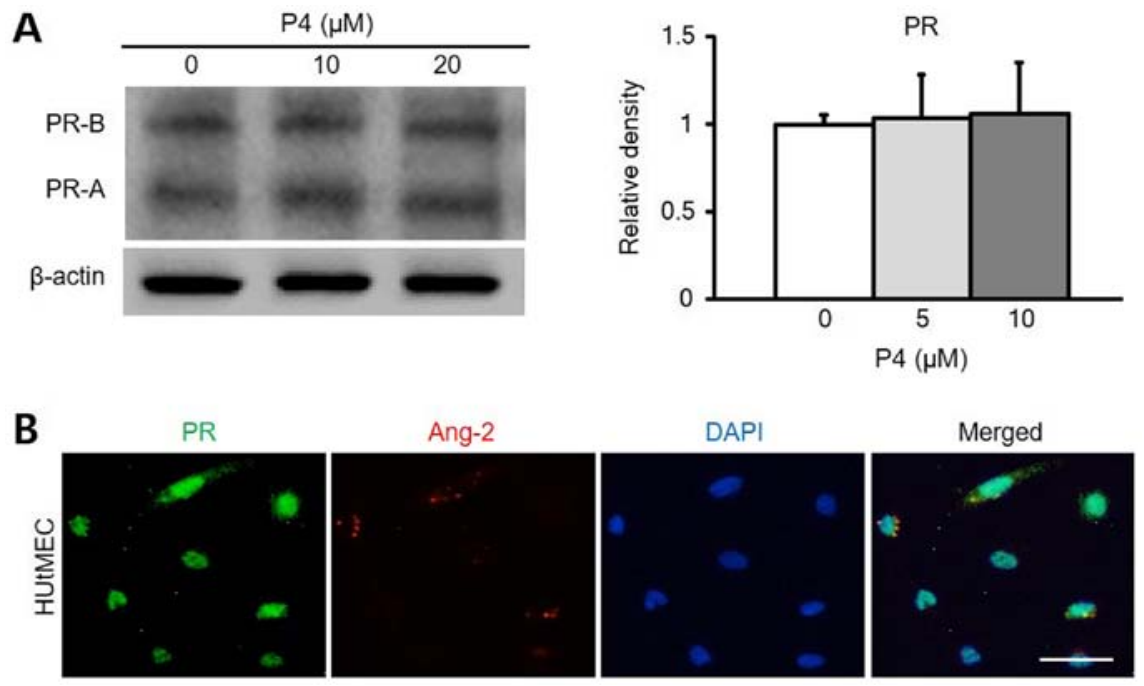

Figure 4. Ang-2 expression is associated with PR in HUtMECs. (A) Comparison of PR protein levels and relative density (right graph) in HUtMECs treated with control and P4 $(5,10$ and $20 \mu \mathrm{M})$. Loading of similar amounts of protein for each sample was verified by a similar intensity of $\beta$-actin and relative density was determined according to the control level. (B) Immunofluorescence staining of PR and Ang-2. Nuclei were counterstained with DAPI (blue). Scale bar, $100 \mu \mathrm{m}$. Ang-2, angiopoietin-2; HUtMECs, human uterine microvascular endothelial cells; P4, progesterone; PR, progesterone receptor
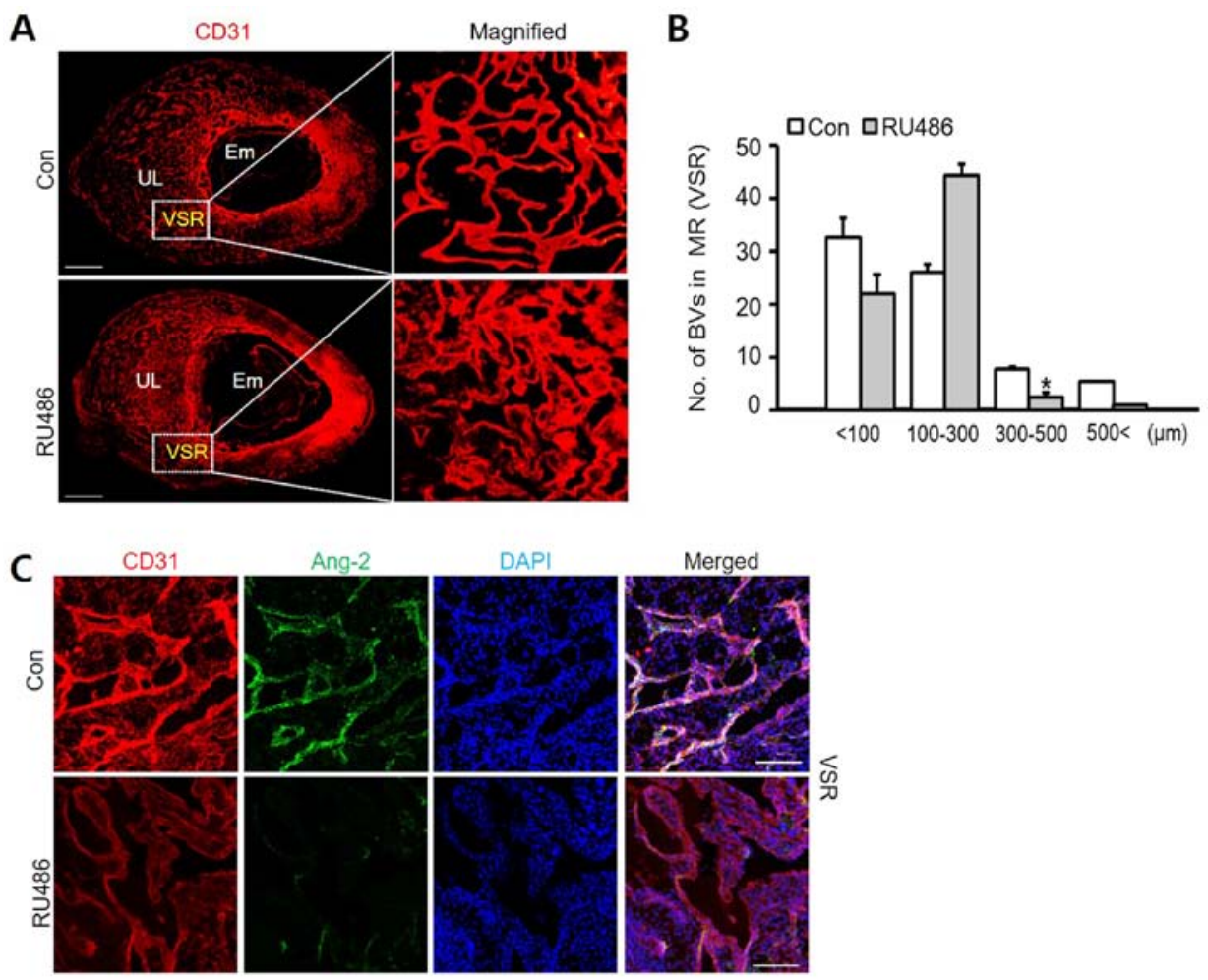

Figure 5. Vascular remodeling in VSR is decreased by RU486. (A) Images showing CD31+ BVs in the uterus at 8.5 dpc following treatment with Con and RU486 (8 mg/kg). $\mathrm{n}=4$. Scale bar, $500 \mu \mathrm{m}$. (B) Number of BVs in MR (VSR) was dependent on their size, compared with Con and treatment RU486. $\mathrm{n}=4$. ${ }^{*} \mathrm{P}<0.02$ vs. Con (t-test). (C) Images showing CD31 ${ }^{+} \mathrm{BVs}$ and Ang-2 in the VSR at $8.5 \mathrm{dpc}$ after treatment with RU486 ( $\left.8 \mathrm{mg} / \mathrm{kg}\right) . \mathrm{n}=4$. Scale bar, $100 \mu \mathrm{m}$. Ang-2, angiopoietin-2; BVs, blood vessels; Con, control; dpc, days post coitum; UL, uterine lumen; Em, embryo; MR, mesometrial region; VSR, venous sinus region.

changes to accept the blood from the mother. In the case of failed transformation, miscarriage and preeclampsia, which are result from vascularity in the uterus, lead to an unsuccessful delivery. Therefore, vascular remodeling in early pregnancy is essential for embryonic growth.

Recently, research has shown that progesterone regulates uterine angiogenesis and vascular remodeling via VEGF-A/ VEGFR-2 signaling in the AMR during pregnancy (1).
However, spatiotemporal vascular remodeling by progesterone in the MR is not fully understood. Interestingly, it is known that endothelial cell proliferation is due to progesterone (29). Thus, in this study, we focused on spatiotemporal ang-2 expression and vascular remodeling in the MR including the VSR during early pregnancy. Furthermore, we investigated the relationship between Ang-2 expression and progesterone treatment through in vitro experiments. 
Our results demonstrated that Ang-2 steadily increases after implantation until placentation. Remarkably, Ang-2 was expressed spatiotemporally in the uterus - especially in the VSR of the MR. In this period, Ang-2 also constantly rose in terms of the total level of protein and mRNA in uterus. Other studies indicated that the amount of mRNA of Ang-2 increased during early pregnancy (23). From this result, we predicted Ang-2 has an effect on vascular remodeling in VSR where lots of blood vessels are lined up by endothelial cells. We found out the correlation between Ang-2 and endothelial cell by performing an X-gal stain. Based on these results, the treatment of HUtMEC with progesterone is very different compared to treatment with progesterone in vitro. Progesterone increased the expression of Ang-2. Progesterone binds to a PR to activate its mechanism. As shown in Fig. 4, PR expressed in the HUtMEC and PR expression was not dose regulated by progesterone concentration. Expression of PR is not related to progesterone level but other factors. According to another study, PR is up-regulated by estrogen at the level of gene expression (30). It seems that the concentration of progesterone has no influence on the expression of $\mathrm{PR}$ in HUtMEC.

Progesterone is a biomarker for decidualization of pregnant uterus (31). Ang-2 was observed in MR during this period. Therefore, we tested the relationship between Ang-2 and PR in decidual angiogenesis using PR blockade named RU486. We injected it intraperitoneally in pregnant mice to observe vascular remodeling during pregnancy. RU486 inhibited Ang-2 mediated vascular remodeling in the MR. Our results indicated that vascular remodeling and decidual angiogenesis are related to signaling pathways that are affected differently by progesterone and Ang- 2 mediated vascular remodeling. Notably, vascular remodeling induced by Ang-2 expression was not dependent on VEGF-A-related signaling in the MR. VEGF-A was mainly expressed in the AMR and was not detected in the VSR during pregnancy (1). Thus, vascular remodeling mediated by Ang-2 signaling would be different to VEGF-A/VEGFR-2 signaling in the pregnant uterus.

Taking all of this information into account, we speculate that the expression of Ang-2 is stimulated by progesterone which influences spatiotemporally different parts of the uterus in early pregnancy. Progesterone most likely induces vascular remodeling via VEGF-A/VEGFR-2 signaling only in the AMR. In the MR, Ang-2 expression by progesterone affects vascular remodeling for placentation.

In conclusion, our results provide insight on previously undefined features of vascular remodeling in the pregnant uterus by Ang-2-mediated signaling during early pregnancy. In the post-implantation period, the pregnant uterus undergoes profound vascular remodeling in response to progesterone for placentation, which has spatiotemporally different effects on the AMR and MR. Progesterone-PRregulated Ang-2 is a key regulator for placentation and prevention of pregnancy failure through spatiotemporal vascular remodeling in the MR.

\section{Acknowledgements}

Not applicable.

\section{Funding}

The present study was supported by the National Research Foundation of Korea funded by the Ministry of Science, ICT and Future Planning (grant no. 2015R1A1A1A05001546 to JWS).

\section{Availability of data and materials}

The datasets used and/or analyzed during the present study are available from the corresponding author on reasonable request.

\section{Authors' contributions}

YGP and JC designed and performed the experiments, analyzed the data, generated the figures and wrote the manuscript. JWS designed, organized and supervised the project, and wrote the manuscript. All authors read and approved the final manuscript.

\section{Ethics approval and consent to participate}

All experiments were performed following approval from the Institutional Animal Care and Use Committees (IACUC) of Jeonbuk National University.

\section{Patient consent for publication}

Not applicable.

\section{Competing interests}

The authors declare that they have no competing interests.

\section{References}

1. Kim M, Park HJ, Seol JW, Jang JY, Cho YS, Kim KR, Choi Y, Lydon JP, Demayo FJ, Shibuya M, et al: VEGF-A regulated by progesterone governs uterine angiogenesis and vascular remodelling during pregnancy. EMBO Mol Med 5: 1415-1430, 2013.

2. Cha J, Sun X and Dey SK: Mechanisms of implantation: Strategies for successful pregnancy. Nat Med 18: 1754-1767, 2012.

3. Wang H and Dey SK: Roadmap to embryo implantation: Clues from mouse models. Nat Rev Genet 7: 185-199, 2006.

4. Smith SD, Choudhury RH, Matos P, Horn JA, Lye SJ, Dunk CE, Aplin JD, Jones RL and Harris LK: Changes in vascular extracellular matrix composition during decidual spiral arteriole remodeling in early human pregnancy. Histol Histopathol 31: 557-571, 2016.

5. Bany BM and Cross JC: Post-implantation mouse conceptuses produce paracrine signals that regulate the uterine endometrium undergoing decidualization. Dev Biol 294: 445-456, 2006.

6. Jones RL and Critchley HO: Morphological and functional changes in human endometrium following intrauterine levonorgestrel delivery. Hum Reprod 15 (Suppl 3): 162-172, 2000.

7. Osol G and Moore LG: Maternal uterine vascular remodeling during pregnancy. Microcirculation 21: 38-47, 2014.

8. Kiepiela P, Smith AN and Rosenberg E: Retraction notice to 'Laboratory markers associated with progression of HIV infection [Best Pract Res Clin Obstet Gynaecol 19: 243-254, 2005]'. Best Pract Res Clin Obstet Gynaecol 21: 883, 2007.

9. Conneely OM, Mulac-Jericevic B and Lydon JP: Progesteronedependent regulation of female reproductive activity by two distinct progesterone receptor isoforms. Steroids 68: 771-778, 2003.

10. Carson DD, Bagchi I, Dey SK, Enders AC, Fazleabas AT, Lessey BA and Yoshinaga K: Embryo implantation. Dev Biol 223: 217-237, 2000.

11. Schumacher A, Costa SD and Zenclussen AC: Endocrine factors modulating immune responses in pregnancy. Front Immunol 5: 196,2014 
12. Wetendorf $\mathrm{M}$ and DeMayo FJ: The progesterone receptor regulates implantation, decidualization, and glandular development via a complex paracrine signaling network. Mol Cell Endocrinol 357: 108-118, 2012.

13. Groothuis PG, Dassen HH, Romano A and Punyadeera C: Estrogen and the endometrium: Lessons learned from gene expression profiling in rodents and human. Hum Reprod Update 13: 405-417, 2007.

14. Saraswati S, Kumar S and Alhaider AA: $\alpha$-santalol inhibits the angiogenesis and growth of human prostate tumor growth by targeting vascular endothelial growth factor receptor 2-mediated AKT/mTOR/P70S6K signaling pathway. Mol Cancer 12: 147, 2013.

15. Bauer SM, Bauer RJ and Velazquez OC: Angiogenesis, vasculogenesis, and induction of healing in chronic wounds. Vasc Endovascular Surg 39: 293-306, 2005.

16. Abbas OL, Borman H, Terzi YK, Terzi A, Bayraktar N and Yazıcı AC: The Notch pathway is a critical regulator of angiogenesis in a skin model of ischemia. Vasc Med 20: 205-211, 2015.

17. Koga K, Osuga Y, Tsutsumi O, Yano T, Yoshino O, Takai Y, Matsumi H, Hiroi H, Kugu K, Momoeda M, et al: Demonstration of angiogenin in human endometrium and its enhanced expression in endometrial tissues in the secretory phase and the decidua. J Clin Endocrinol Metab 86: 5609-5614, 2001.

18. Augustin HG, Koh GY, Thurston G and Alitalo K: Control of vascular morphogenesis and homeostasis through the angiopoietin-Tie system. Nat Rev Mol Cell Biol 10: 165-177, 2009.

19. Carmeliet P: Angiogenesis in health and disease. Nat Med 9: 653-660, 2003.

20. Chung AS and Ferrara N: Developmental and pathological angiogenesis. Annu Rev Cell Dev Biol 27: 563-584, 2011.

21. Fan X, Krieg S, Kuo CJ, Wiegand SJ, Rabinovitch M, Druzin ML, Brenner RM, Giudice LC and Nayak NR: VEGF blockade inhibits angiogenesis and reepithelialization of endometrium. FASEB J 22: 3571-3580, 2008.

22. Kwak HJ, So JN, Lee SJ, Kim I and Koh GY: Angiopoietin-1 is an apoptosis survival factor for endothelial cells. FEBS Lett 448 249-253, 1999.
23. Matsumoto H, Ma WG, Daikoku T, Zhao X, Paria BC, Das SK, Trzaskos JM and Dey SK: Cyclooxygenase-2 differentially directs uterine angiogenesis during implantation in mice. J Biol Chem 277: 29260-29267, 2002

24. Felcht M, Luck R, Schering A, Seidel P, Srivastava K, Hu J, Bartol A, Kienast Y, Vettel C, Loos EK, et al: Angiopoietin-2 differentially regulates angiogenesis through TIE2 and integrin signaling. J Clin Invest 122: 1991-2005, 2012.

25. Geva E, Ginzinger DG, Moore DH II, Ursell PC and Jaffe RB: In utero angiopoietin-2 gene delivery remodels placental blood vessel phenotype: A murine model for studying placental angiogenesis. Mol Hum Reprod 11: 253-260, 2005.

26. Tsuzuki T, Okada H, Cho H, Shimoi K, Miyashiro H, Yasuda K and Kanzaki H: Divergent regulation of angiopoietin-1, angiopoietin-2, and vascular endothelial growth factor by hypoxia and female sex steroids in human endometrial stromal cells. Eur J Obstet Gynecol Reprod Biol 168: 95-101, 2013.

27. Akwii RG, Sajib MS, Zahra FT and Mikelis CM: Role of Angiopoietin-2 in Vascular Physiology and Pathophysiology. Cells 8: 471, 2019.

28. Cartwright JE, Fraser R, Leslie K, Wallace AE and James JL: Remodelling at the maternal-fetal interface: Relevance to human pregnancy disorders. Reproduction 140: 803-813, 2010.

29. Walter LM, Rogers PA and Girling JE: The role of progesterone in endometrial angiogenesis in pregnant and ovariectomised mice. Reproduction 129: 765-777, 2005.

30. Ing $\mathrm{NH}$ and Tornesi MB: Estradiol up-regulates estrogen receptor and progesterone receptor gene expression in specific ovine uterine cells. Biol Reprod 56: 1205-1215, 1997.

31. Das A, Mantena SR, Kannan A, Evans DB, Bagchi MK and Bagchi IC: De novo synthesis of estrogen in pregnant uterus is critical for stromal decidualization and angiogenesis. Proc Natl Acad Sci USA 106: 12542-12547, 2009.

(i) $\Theta$ This work is licensed under a Creative Commons Attribution-NonCommercial-NoDerivatives 4.0 International (CC BY-NC-ND 4.0) License. 